\title{
Efficacy of rigid-pole exercise training for spinal mobility
}

Keywords: rigid-pole exercise training, range of motion, spinal mouse, mobility, spiral motion

\section{Commentary}

Physical medicine has been recently more important in the actual clinical practice. Various athletes and patients with diseases have been treated with medical agents. ${ }^{1}$ Furthermore, application of adequate rehabilitation can improve nervous system stimulation, muscle power training, muscle stretching, and muscle flexibility. ${ }^{2}$ Then, continuing these practice will be sure to bring better physical condition such as standing posture, smooth walking, relaxed movements, and increased range of motion (ROM).

The purpose of this article includes some topics of spinal stability/ mobility, Spinal Mouse, pole exercise and the usefulness of the rigid pole. It is the stability of the spine that plays a central role in the functioning of the human body. ${ }^{3}$ This involves three important elements. They are nervous system (control), muscle (active) and bone (passive). ${ }^{4}$ The muscles can always adjust for the maintenance and movements for human body. From global point of view, some muscles are involved in the regulation, such as the rectus abdominis (RA), lateral oblique (EO) and medial oblique (IO). In contrast, local regulating muscles include the transversus abdominis (TrA), multifidus (MF) and interspinalis situated in deeper trunk. ${ }^{3,4}$

For the actual practice and research, spinal stability/mobility have been crucial factors. In order to evaluate them, SpinalMouse ${ }^{{ }^{5}}{ }^{5}$ can be used. ${ }^{6}$ It has been known as a useful non-invasive device, and can measure the detail shape and mobility of the spine. ${ }^{7}$ It can check the data of the spinal curvature in the sagittal and frontal planes. Furthermore, we can evaluate spine function associated with trunk posture and gait characteristics. ${ }^{8}$

As a study of Spinal Mouse, the importance of lumbar multifidus (LM) muscle has been reported. LM shows a stronger function than lumbar extension. ${ }^{9}$ In addition, LM action for lumbar facet joint adaptation and lumbar flexion are also important, which is involved in lumbar stability and low back pain (LBP). ${ }^{9}$

From the study of Spinal Mouse on LBP, the stiffness degree of the LM was recognized to be crucial and significant factor. ${ }^{10}$ Further, Spinal Mouse clarified the difference between characteristic craniofacial morphology and thoracic-lumbar-sacral spine inclination. ${ }^{11}$

Authors have continued research on Spinal Mouse so far. Dysfunction of LM in asymmetry/muscle thickness reduction was observed associated with LBP and thoracic flexibility/stability. ${ }^{12}$ Consequently, effect of exercise for lower thorax was found on the LM and spinal flexion mobility. ${ }^{12}$ Recently we investigated the range of motion (ROM) of thoracic cage after the intervention of pole exercise. ${ }^{13}$ The pole exercise was performed and compared between on the shoulder and at the armpit. ${ }^{14}$ As the result, the former showed larger ROM tendency. ${ }^{13}$

Concerning pole exercise, the authors have conducted rigid-pole exercise training. The author Murakami has taken care of Tokyo

\author{
Volume 4 Issue 5 - 2019
}

\author{
Hiroshi Bando, ${ }^{1,2}$ Akito Moriyasu, ${ }^{3,4}$ Mitsuru \\ Murakami, ${ }^{5}$ \\ 'Medical Research, Tokushima University, Japan \\ 2Japan Masters Athletics, Tokushima division, Japan \\ ${ }^{3}$ Rehabilitation Research Group for body and heart in Shikoku, \\ Japan \\ ${ }^{4}$ Akiboshi Bright Star Training Rehabilitation Center, Japan \\ 5Japan Masters Athletics, Kagawa division, Japan
}

Correspondence: Hiroshi Bando, Medical Research, Tokushima University, Nakashowa I-6I, Tokushima 770-0943, Japan, Tel +8I90-3187-2485, Email pianomed@bronze.ocn.ne.jp

Received:September 23, 2019 | Published: September 27, 2019

Paralympic athletes and continues to give guidance at workshops for Masters athletes how to run safe and fast. ${ }^{15,16}$ Furthermore, rigid-pole training using bamboo has been introduced and more prevalent. ${ }^{14}$ The author Moriyasu continues to teach professional baseball and soccer players as well as medical rehabilitation for patients with various diseases. Further, Moriyasu pole exercise training has been advocated and spread until now.

The detail methods of the pole exercise training are shown as follows. ${ }^{14,15}$ As the device, everyone can use bamboo pole, Moriyasu pole or other rigid-pole. Moriyasu pole has been useful for a variety of movements. The reason is that the pole consists of 4 parts (Figure 1). Anyone can connect them easily, where the pole become from 80 $\mathrm{cm}$ to $160 \mathrm{~cm} .{ }^{17}$ Thus, any movement can be performed using the pole.

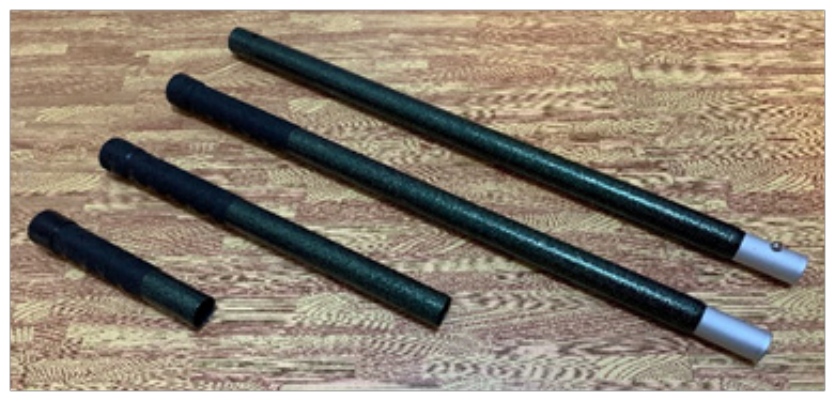

Figure I Moriyasu Pole.

The length of the pole can be $80,100,140,160 \mathrm{~cm}$ by the connection.

Standard pole exercise training has its fundamental six main movements in our protocol. ${ }^{14}$ They are i) lateral bending, ii) axis rotation, iii) wave motion, iv) backward spiral, v) forward spiral and vi) warp \& rounding. ${ }^{14}$ Among them, two movements were shown in Figure 2. The movement i) can be performed using $60 \mathrm{~cm}$ (Figure 2 A), and the movements iv) and v) can be performed using $160 \mathrm{~cm}$ (Figure 2B). The person in charge of the exercise in Figure 2 is second author Moriyasu, where we have informed consent concerning this situation. 

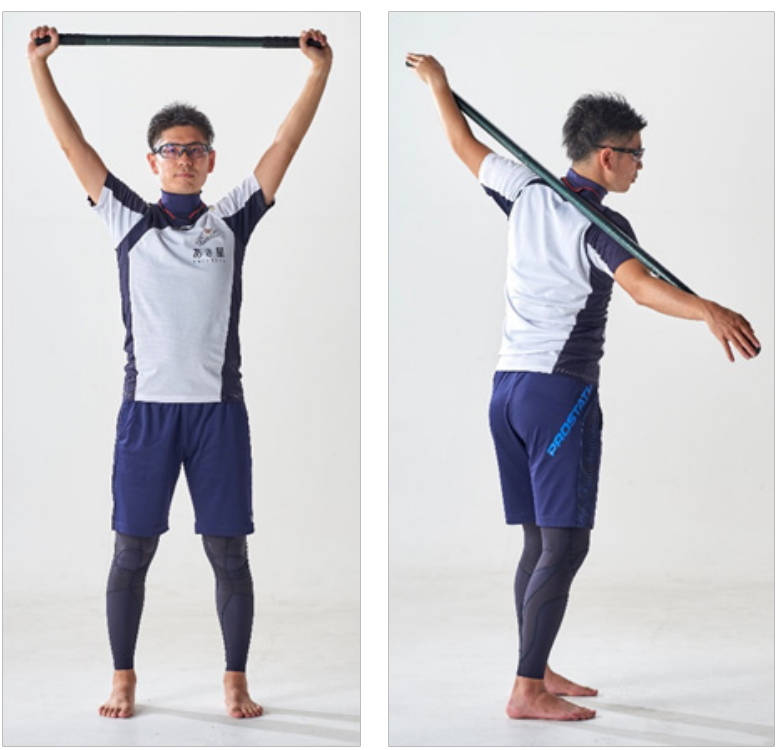

Figure 2 Performance of pole exercise training using Moriyasu pole.

Figure 2A lateral bending movement

Figure 2B forward and backward spiral movement

The length of the pole can be $80,100,140,160 \mathrm{~cm}$ by the connection.

The length of the pole in Figure $2 \mathrm{a}$ and $2 \mathrm{~b}$ were $80 \mathrm{~cm}$ and $160 \mathrm{~cm}$, respectively.

After subjects receive these 6 basic movements of pole exercise, it is necessary to evaluate the response or effect in the light of physical mobility and exercise function $[14,17]$. We have chosen adequate examinations including 7 physical mobility tests. They are 1) finger floor distance (FFD), 2) straight leg raise (SLR), 3) shoulder extension test (SET), 4) body warp prone position, 5) heel buttock distance (HBD), 6) Thomas test and 7) Wing test. ${ }^{14,17}$

There 7 tests show the correlation with 4 different portion of the vertebrae. They are a) upper thoracic vertebrae: $1,2,3,4)$, b) lower thoracic vertebrae: $1,2,3,4)$, c) lumbar vertebrae: $4,5,6,7)$, d) sacroiliac joint: $1,2,4,5,6,7)$. As vertebrae function changes, physical mobility changes. After that, exercise function will change. ${ }^{14,17}$ Thus, the efficacy of the pole exercise training would be evaluated by several physical mobility tests.

From mentioned above, these 7 tests are recommended to check when a patient or subject shows spinal-related health problems. After investigating the cause and details, treatment and care will be given. In that case, practical training of pole exercise training would be one of the options for expecting the efficacy. ${ }^{14,17}$ As for pole exercise, there was a related previous report. ${ }^{18}$ The activity of trunk muscle was compared with rigid-pole and non-rigid pole, in which the latter was predominant in the activation of trunk muscles. ${ }^{18}$ Successively, increasing coordination and balance skills were investigated. ${ }^{19}$

In summary, some topics were presented in this commentary with spinal mobility in the subjects with various situations. Furthermore, we added some research of Spinal Mouse for evaluation of thoracic and lumbar vertebrae. Authors have continued practice and teaching pole exercise training with effective for improving spinal mobility. This report would be expected to become reference data for future research development.

\section{Funding}

None

\section{Acknowledgements}

None.

\section{Conflicts of interest}

The author declares no conflict of interest.

\section{References}

1. Grecheneva AV, Dorofeev NV, Kuzichkin OR. The Technique of the optimization of the process of the rehabilitation of the spine based on the individual information model of the spine. 2019 International Science and Technology Conference. 2019.

2. Spencer S, Wolf A, Rushton A. Spinal-exercise prescription in sport: classifying physical training and rehabilitation by intention and outcome. Journal of Athletic Training. 2016;51(8):613-628.

3. Panjabi M. Clinical spinal instability and low back pain. J Electromyogr Kinesiol. 2003;13:371-379.

4. Izzo R, Guarnieri G, Guglielmi G, et al. Biomechanics of the spine. Part I: spinal stability. European Journal of Radiology. 2013;82:118-126.

5. Demarteau J, Jansen B, Van Keymolen B, et al. Trunk inclination and hip extension mobility, but not thoracic kyphosis angle, are related to 3D-accelerometry based gait alterations and increased fall-risk in older persons. Gait \& Posture. 2019;72:89-95.

6. Roghani T, Khalkhali Zavieh M, Rahimi A, et al. The reliability of standing sagittal measurements of spinal curvature and range of motion in older women with and without hyperkyphosis using a skin-surface device. J Manipul Physiol Ther. 2017;40:685-691.

7. Livanelioglu A, Kaya F, Nabiyev V, et al. The validity and reliability of "spinal mouse" assessment of spinal curvatures in the frontal plane in pediatric adolescent idiopathic thoraco-lumbar curves. European Spine Journal. 2015;25(2):476-482.

8. Merchant RA, Banerji S, Singh G, et al. Is trunk posture in walking a better marker than gait speed in predicting decline in function and subsequent frailty?. J Am Med Dir Assoc. 2016;17:65-70.

9. Norris C, Matthews M. The role of an integrated back stability program in patients with chronic low back pain. Complementary Therapies in Clinical Practice. 2008;14:255-263.

10. Masaki M, Ikezoe T, Yanase K, et al. Association of pain history and current pain with sagittal spinal alignment and muscle stiffness and muscle mass of the back muscles in middle-aged and elderly women. Clin Spine Surg. 2019;32(7):E346-E352.

11. Piancino MG, Dalmasso P, Borello F, et al. Thoracic-lumbar-sacral spine sagittal alignment and cranio-mandibular morphology in adolescents. Journal of Electromyography and Kinesiology. 2019;48:169-175.

12. Kurihara R, Ozaki J, Dakeshita T, et al. Effects of exercise therapy for lower thorax on lumbar multifidus and spinal flexion mobility. J Jap Phys Ther Assoc. 2016;44(s2):P-MT-12-3.

13. Kurihara R, Fujimoto D, Dakashita T, et al. The influence of Pole exercise on the range of motion of thoracic spine. Clinical Research in Orthopaedics. 2019;2(1):1-5.

14. Moriyasu A, M Murakami, Bando H. Pole Exercise--simple way to anyone, changing the standard of health. Medical Information Service; Tokyo, Japan.

15. Murakami M, Bando H, Moriyasu A. Flexibility of the chest-lumbar region in athletic athletes. Int Phys Med Rehab J. 2019;4(5):207-208.

16. Bando H, Murakami M, Moriyasu A. Development of health and medical research for long similar to successful marathon running. $J$ Health Med Res. 2019;1(1):3. 
17. Moriyasu A, Bando H, Akayama R, et al. Thorax flexibility can be increased by standing pole exercise. Int J Phys Med Rehabil. 2017;6:444.

18. Chung JS, Park S, Kim J, et al. Effects of flexi-bar and non-flexi-bar exercises on trunk muscles activity in different postures in healthy adults. J Phys Ther Sci. 2015;22727(7):2275-2278.
19. Lee SJ, Kim YN, Lee DK. The effect of flexi-bar exercise with vibration on trunk muscle thickness and balance in university students in their twenties. J Phys Ther Sci. 2016;28(4):1298-302. 